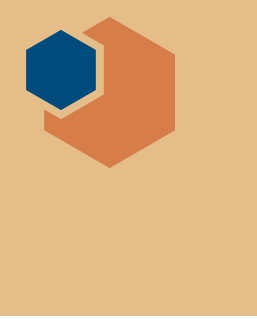

\section{US-Brazil Science and Technology Cooperation highlights nanotechnology}

\begin{abstract}
A a recent United States-Brazil Joint Commission Meeting on Science and Technology Cooperation in Brasilia, Brazil, representatives from Brazil and the United States discussed several areas of current collaboration, such as natural disaster management and ocean science. In addition, Brazil proposed nanotechnology as one of three new areas of bilateral engagement. Individual research groups in the United States and Brazil have several productive collaborations related to nanomaterials, nanocomposites, and nanometrology, and designating nanotechnology as a formal area of cooperation could lead to greater
\end{abstract}

Obama welcomed the Joint Commission's action plan and the discussion of a more formal commitment to nanotechnology collaborations.

It is not yet clear what a formal nanotechnology collaboration would look like, but representatives from the Brazilian Ministry of Science, Technology, and Innovation; Brazilian National Institute of Metrology, Quality and Technology; and the US National Nanotechnology Initiative began exploring shared national strategies and discussing access to user facilities at a recent meeting. The US National Science Foundation (NSF) Nanoscale Science and Engineer-

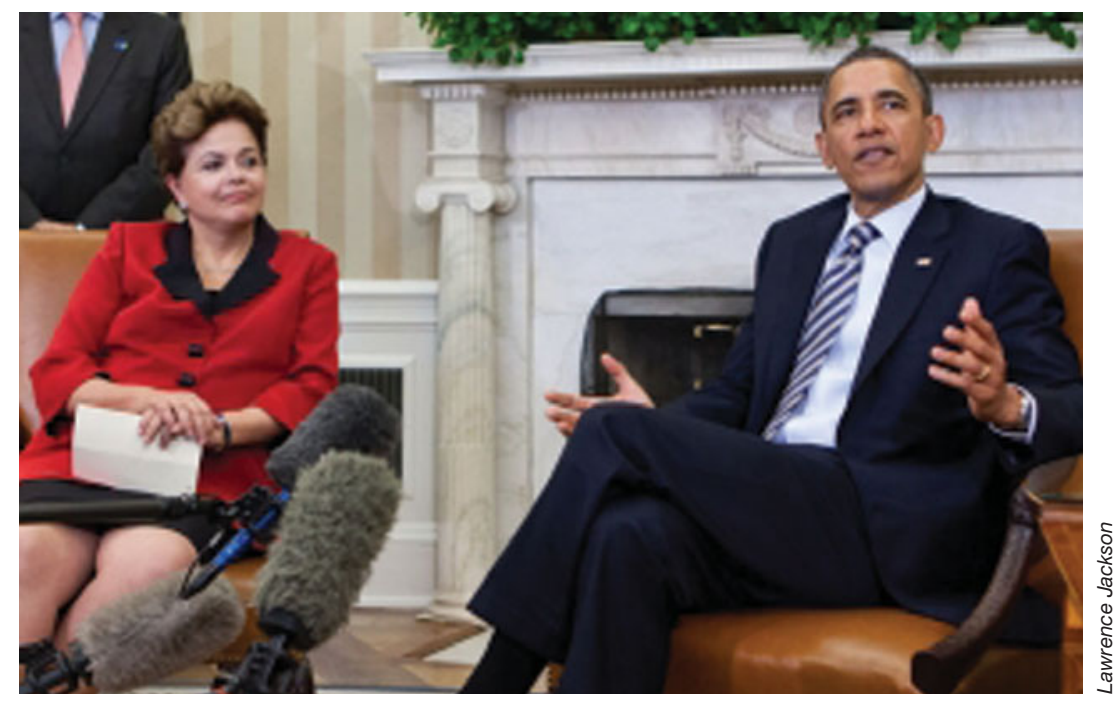

President Barack Obama (right) and President Dilma Rousseff of Brazil in the Oval Office, April 9, 2012.

access to user facilities and support for scientist exchange programs, among other benefits.

During an April visit to the United States, Brazilian President Dilma Rousseff highlighted the importance of the ongoing collaborative science efforts, and along with US President Barack ing Centers and the Brazilian Nanotechnology Centers are exploring student exchanges and other collaborative opportunities.

Collaborations like these would be beneficial for both the United States and Brazil, according to Adalberto Fazzio, Nanotechnologies Coordinator for the
Ministry of Science, Technology, and Innovation in Brazil. In addition to the new contacts and opportunities that arise from student exchanges and access to user facilities, Fazzio said that Brazil hopes to learn from the United States about effective nanotechnology policies and national investment strategies, as well as benefit from US expertise on linking academia and industry. Brazil has a lot to offer the United States in terms of its human capital and industrial opportunities.

Brazil has a very active nanoscience community that engages around 2500 scientists. Within Brazil, nanoscience and nanotechnology are the primary focus of eight national laboratories, 16 national science and technology institutes created at existing laboratories with federal funding, and several themed cooperative networks that receive federal funding and are composed of multiple researchers at various institutions. The Brazilian government recently created an Interministerial Nanotechnology Committee, charged with integrating the strategies of the different ministries that fund nanotechnology-related efforts.

Brazilian federal agencies plan to invest about R\$100 million (USD\$49 million) in nanotechnology research per year for the next five years. According to Carlos Achete, director of the Materials Metrology Division of Inmetro (Brazil's National Institute of Metrology), these investments are driven by a desire to build national industry and grow the economy, but also to meet critical international challenges like the rising demand for energy. Much of Brazil's nanotechnology research is also motivated by industrial needs. Among the companies that do nanotechnology research and development, the largest segment is focused on nanocomposites and materials, with other common areas including pharmaceuticals, energy, health, and biotechnology.

Brazil was the first Latin American country to implement public support for nanotechnology programs, starting with an investment in four national research networks in 2001. Since the beginning, the government's nanotechnology 
investment has largely been in networks that coordinate the work of many researchers across the country, with some international partners, in order to boost the country's industrial competitiveness without huge investments in infrastructure. This positions Brazil well for international partnerships that require virtual collaborations, short-term researcher exchanges, and collaborations among people with different cultures.

Currently, the Brazilian government is in the midst of a four-year effort to facilitate new international collaborations and supplement educational experiences for students through study-abroad scholarships. Under the Science Without Borders program, some 75,000 students and postdoctoral researchers will receive scholarships from the Brazilian government to study abroad, and another 26,000 are receiving similar scholarships from Brazilian companies. Approximately 20,000 of the participants are expected to study in the United States, with the others studying in France, Germany, Italy, and the United Kingdom.

In March 2011, President Obama announced a similar program, 100,000 Strong in the Americas. This program aims to increase the number of US students studying in Latin America and the Caribbean to 100,000 , and the number of Latin American students studying in the United States to 100,000 . Through Science Without Borders and 100,000 Strong in the Americas, the United States and Brazil aim to strengthen institutional partnerships, facilitate new collaborations, equip the next-generation workforce, and increase prosperity within the region.

The United States and Brazil have collaborated on a number of science and technology efforts during the last few decades. Many of these efforts have been facilitated by the Joint Commission on Science and Technology Cooperation. The Joint Commission was established by a 1984 agreement between the United States and Brazil to plan and coordinate cooperative science and technology efforts that advance the state of science, raise the level of technology, and contribute to attaining common goals.

Kendra Redmond
EU-funded projects go public www.openaire.eu

$\mathbf{T}$ he European Commission (EC) wants results from European Union's (EU) Seventh Framework Program (FP7) (2007-2013) and Horizon 2020 (20142020) projects to produce fully "open access" publications. In this effort, the $\mathrm{EC}$ is funding, through FP7, the project "Open access infrastructure for research in Europe" (OpenAIRE). This project will provide a single access point to all the open access publications produced by FP7 projects during the course of the Seventh Framework Program.

"To try and push more open access publishing, the European Commission has made open access publishing mandatory for around $20 \%$ of FP7 projects," said Natalia Manola, the project's manager. "This is written into the contract, but it is still a soft target - hard to enforce and monitor or really measure the impact. The idea of online open access is still quite new and one of the biggest problems is that projects will publish some results in traditional journals and some in open access publications. Knowledge is fragmented and it is difficult to see the output of a project because it is spread around so much. We want everything to be accessible by everyone."
The project's website, www.openaire. eu, contains links to FP7 open access guidelines and a FAQs page to address questions such as how researchers are to follow the guidelines if their publishers do not allow authors to archive their articles.

OpenAIRE is a repository network and is based on technology developed in an earlier project called Driver. The Driver engine trawled through existing open access repositories of universities, research institutions, and a growing number of open access publishers. It would index all these publications and provide a single point of entry for individuals, businesses, or other scientists to search a comprehensive collection of open access resources.

OpenAIRE uses the same underlying technology to index FP7 publications and results. FP7 project participants are encouraged to publish their papers, reports, and conference presentations to their institutional open access repositories. The OpenAIRE engine constantly trawls these repositories to identify and index any publications related to FP7funded projects. Working closely with the EC's own databases, OpenAIRE matches publications to their respective FP7 grants and projects providing a seamless link between these previously separate data sets.

OpenAIRE is also linked to CERN's open access repository for "orphan" publications. Any FP7 participants who do not have access to their own institutional repository can still submit open access publications by placing them in the CERN repository.

The project is collecting usage statistics of the portal and the volume of open access publications. It will provide this information to the Commission and use these data to inform European policy in this domain.

OpenAIRE is working closely to integrate its information with the CORDA database, the master database of all EUfunded research projects.

Aiming to build the "knowledge infrastructure" of the European Research Area, an extension project, OpenAIREplus, is now working in parallel to OpenAIRE to add open access data sets to the mix and create a so-called "information space" where publications, data sets, and funding information (EC and national) are interlinked. Researchers can make their raw data, benchmark data, or data objects associated to publications available for others to analyze or use. 